\title{
Time frequency distributions of TMJ sounds
}

\author{
S.E. WIDMALM, W.J. WILLIAMS* and C. ZHENG* Department of \\ Cariology and General Dentistry and *Department of Electrical Engineering and Computer Science, \\ University of Michigan, Ann Arbor, MI, U.S.A.
}

\section{Summary}

For analysis of time-varying signals such as the TMJ sounds, it is often desirable to know how the frequency components change with time, using methods of timefrequency analysis. The aim of this study was to compare two of the most familiar methods for energy density representation with a newly developed technique. The sounds were recorded with a microphone fastened to the subject's forehead, transformed to the time-frequency domain and displayed as 3D- and contour plots using spectrogram, Wigner distribution (WD), and the reduced interference distribution (RID) to display their time-frequency energy distributions. The spectrogram resolved only the lowfrequency components. The WD provided higher resolution but also exhibited strong interference between components. The RID gave a detailed representation of the TMJ signals' relative energy distribution in the time and frequency domains, with a great reduction in the interference or cross terms. The RID therefore appears to be most useful in the application of time-frequency distributions in classification of TMJ sounds.

\section{Introduction}

It is generally accepted that joint sounds should be recorded during examination of patients with suspected TMJ and/or CM disorders (Ekensten, 1952; Watt, 1966; Farrar, 1978). Sounds may imply pathological conditions, but they do also occur during jaw movements in subjects without any history, signs or symptoms of TMJ pathology (Ramfjord \& Ash, 1983). Various attempts to characterize the TMJ sounds in order to differentiate between those that may indicate pathology and those that do not (Eriksson, Westesson \& Rohlin, 1985; Roberts et al., 1986; Gay \& Bertolami, 1987) have so far not yielded conclusive results that could be used in individual diagnosis. The interpretation of the sound events, their expected frequency ranges and their clinical significance are still controversial issues (Widmer, 1989).

Many studies have recorded the sounds' analogue forms only (Ekensten, 1952; Widmalm \& Larsson, 1982; Eriksson et al., 1985; Isberg, Widmalm \& Ivarsson, 1985). However, it is not possible to interpret some variations in the analogue waveforms without separating the signals into their spectral components. Stationary signals are often described by their power spectra. This approach has also been used for TMJ sounds to show the distribution of the signal energy over a range of frequencies (Oster et al., 1984; Gay \& Bertolami, 1988). However, a disadvantage of conventional power spectra is that completely different analogue signals can have exactly the same spectra (Boashash, 1991).

Correspondence: Dr Sven E. Widmalm, 1565 Kuehnle, Ann Arbor, MI 48103, U.S.A. 
For time-varying signals, such as the electronically recorded TMJ sounds, it is often desirable to know more precisely how their frequency components change with time (Cohen, 1989). This can be achieved by studying their time-frequency distributions (Williams \& Jeong, 1990). The concept of expressing sound in terms of a timefrequency representation has existed in the case of the musical score for centuries. Each note represents the pitch (frequency) of a sound and its duration. Complex musical pieces consist of many notes with distinct pitches and durations. The trained human ear is capable of abstracting these complex relationships and recording them in terms of musical notation. By comparison, previous technological means of computing similar relationships in sound have been crude. It has been considered that a tone must persist for a certain length of time for its frequency to be determined exactly, i.e. the signal must remain stationary for a certain time in order to determine timefrequency relationships using techniques such as the spectrogram or sonogram. This is because a fixed-length time window is moved along the signal, and the frequencies of the tones lying within the window are determined. Thus any variation in the frequency of a component within the window produces a confusing result.

Two of the most familiar methods for displaying a signal's time-frequency distribution are the spectrogram and the Wigner distribution (WD) (Cohen, 1989). The spectrogram has been used for analysis of TMJ sounds by Oullette (1974) and also by Gay \& Bertolami (1987) who, however, concluded that the spectrogram has limited applications in the differential diagnosis of TMJ disorders. The reduced interference-distribution (RID) is a new time-frequency distribution, which is a true conjoint distribution of the energy in a signal in terms of precise time-frequency co-ordinates (Choi \& Williams, 1989; Williams \& Jeong, 1990). It allows a more precise analysis of the true timefrequency components of the signals. The aim of this study was to compare the usefulness of this method with that of the spectrogram and the Wigner distribution, in the analysis of TMJ sounds.

\section{Methods}

TMJ sounds and vibrations were recorded with a heart sound microphone, which measures the rate at which the velocity of the vibrating tissues is changing (ElemaSchönander Heart Sound Microphone EMT 25C)*. The microphone has a resonant frequency of about $1200 \mathrm{~Hz}$ and $3 \mathrm{~dB}$ roll offs at 40 and $1200 \mathrm{~Hz}$, respectively, according to the manufacturer. It was used while fastened to the subject's forehead (Widmalm \& Hedegård, 1974). The sounds were amplified (TECA TE42 system) $\dagger$ with AA6 Mk.III amplifiers. The passband of the filter was $16 \mathrm{~Hz}$ on the low and $16,000 \mathrm{~Hz}$ on the high end. A Metrabyte DAS-16F A/D conversion board $\ddagger$ was used for digitization. The maximal time resolution was $10 \mu$ s and the amplitude resolution was 12 bits. The sampling rate used was $10 \mathrm{kHz}$. The accuracy of the $A / D$ converter was $0.01 \%$ of reading \pm 1 bit. The accuracy of the sampling rate was checked during each session by digitizing a calibration signal (error $\ll 0.5 \%$ ). In signal processing of this type, the number of data points is preferably $2^{n}$. The duration of the analysis window should be as short as possible, and was either $12.8 \mathrm{~ms}$ ( $2^{7}$ samples), $25.6 \mathrm{~ms}$ ( $2^{8}$ samples) or

\footnotetext{
* Siemens Medical Systems, NJ, U.S.A.

$\dagger$ TECA Corp., Pleasantville, NY, U.S.A.

$\ddagger$ MetraByte Corp., Taunton, MA, U.S.A.
} 
$51 \cdot 2 \mathrm{~ms}$ ( $2^{9}$ samples). Time-frequency $(t-f)$ distributions were displayed as $3 \mathrm{D}$ - and contour plots using algorithms for spectrogram, Wigner-distribution and the reduced interference distribution (RID) (Williams \& Jeong, 1990). The input file was a time series of data, and the output was a $128 \times 64 t-f$ representation matrix.

\section{Test results and experience}

The RID was found to resolve the sound waveforms (Fig. 1) into distinct timefrequency components giving a detailed representation of the signals' relative energy distribution in the time and frequency domains (Figs 2 and 3). The spectrogram (Figs 4 and 5) resolved only the low-frequency components. The Wigner distribution (WD) (Figs 6 and 7) provided higher resolution, but also strong interference and crossterms. The RIDs and contour plots of the sound recordings had a frequency range with distinct components up to $5000 \mathrm{~Hz}$.

\section{Discussion}

The use of time-frequency $(t-f)$ distributions makes it possible to observe features of the TMJ sounds that are not observable in the analogue waveforms or in conventional power spectra. For instance, distinct time-frequency components with different timeand frequency ranges can be seen, as shown in Fig. 2. Those components may have different anatomical origins and causes, distinctions that cannot be readily observed without using a $t-f$ distribution such as, for example, the RID. $T-f$ distributions should therefore have important potential applications in visual and computer-aided classification of consistent TMJ sounds. By using them it should be possible to identify and map in two dimensions features such as location, amplitude and duration of energy peaks. Such 'maps' can then be used for computer-aided classification with programs for pattern recognition.

Several possible methods can be used. This study has demonstrated that, among the principal methods, the RID has several advantages, yielding more details than the spectrogram, and with a great reduction of the interference terms or cross-terms which occur in the Wigner distributions. The RID therefore appears to be the method of choice when using $t-f$ distributions for the classification of TMJ sounds.

One reason for the more accurate and detailed results that are obtained with RID,
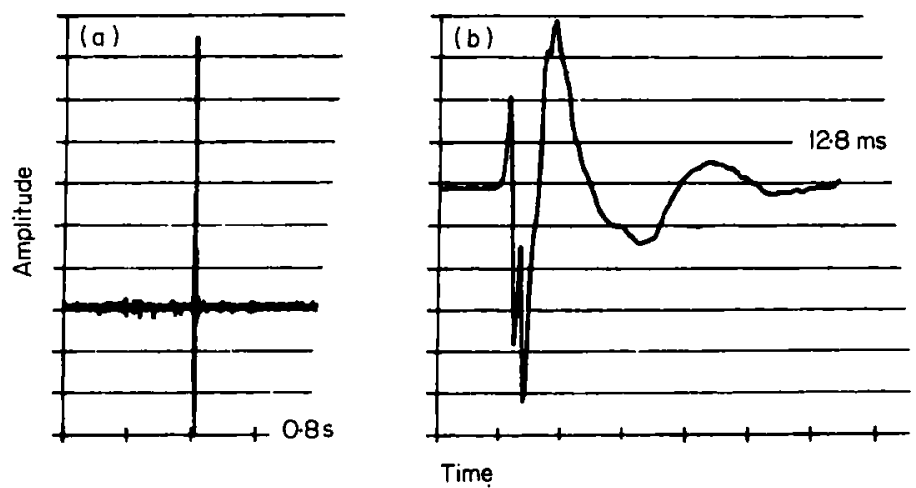

Fig. 1. Analogue form of a TMJ clicking, during opening, displayed with (a) low and (b) high time resolution. 


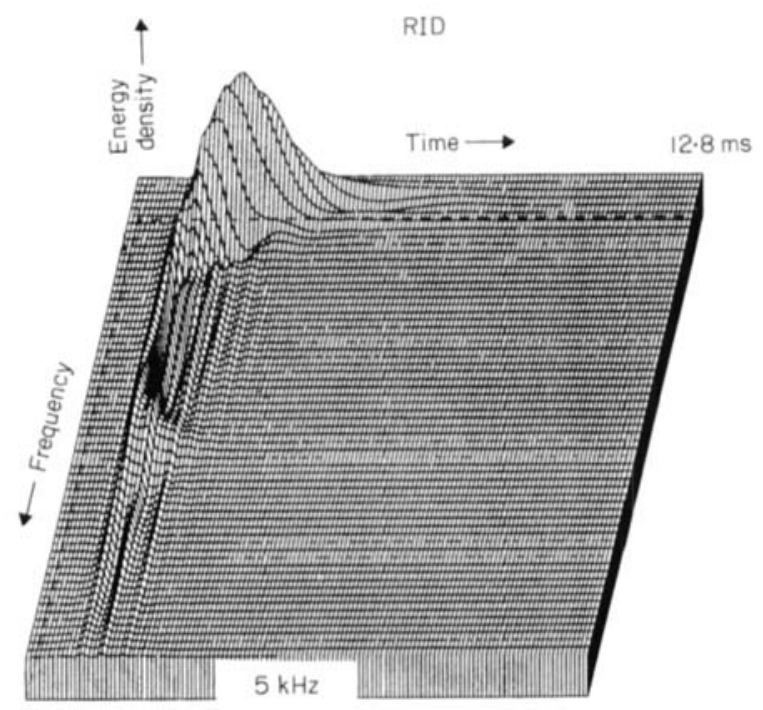

Fig. 2. Reduced interference distribution (RID); three-dimensional plot of the same clicking as that shown in Fig. 1. There are two distinct time-frequency components that occur almost simultaneously in time, but are clearly separated in the frequency range. A possible explanation is that during reduction, the condylar head first hits the disc and then, a few ms later, the condylar head and the disc together impact on the articular eminence. The sharp ridges parallel to the frequency axis are due to the sharp peaks in the sound signal. Sharp peaks are composed of a broad complement of components over a wide band of frequencies. The ridges may be caused by the condyle and/or the disc sliding over an irregular surface.

RID contour plot

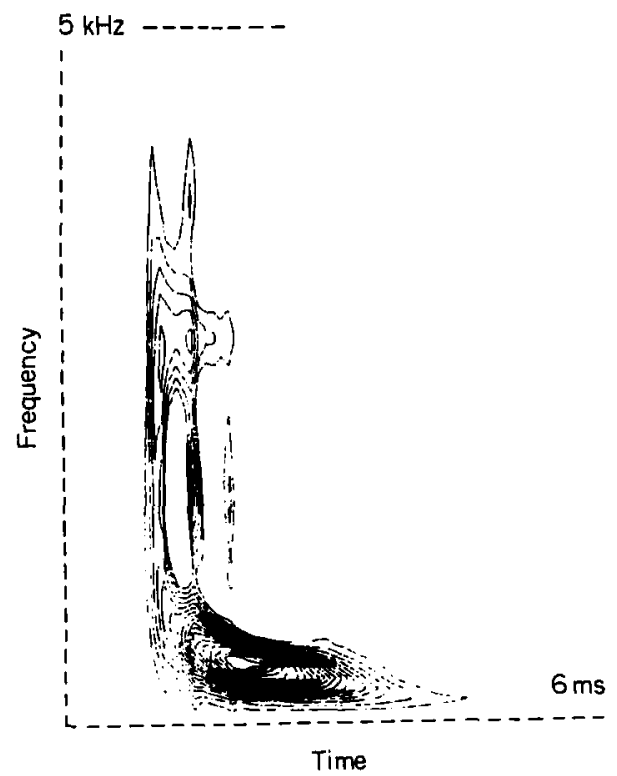

Fig. 3. RID contour-plot of the temporomandibular joint (TMJ) clicking sound shown in Fig. 1. Each line represents a step of increased signal energy, in the same way as a contour map of a landscape represents differences in height. 


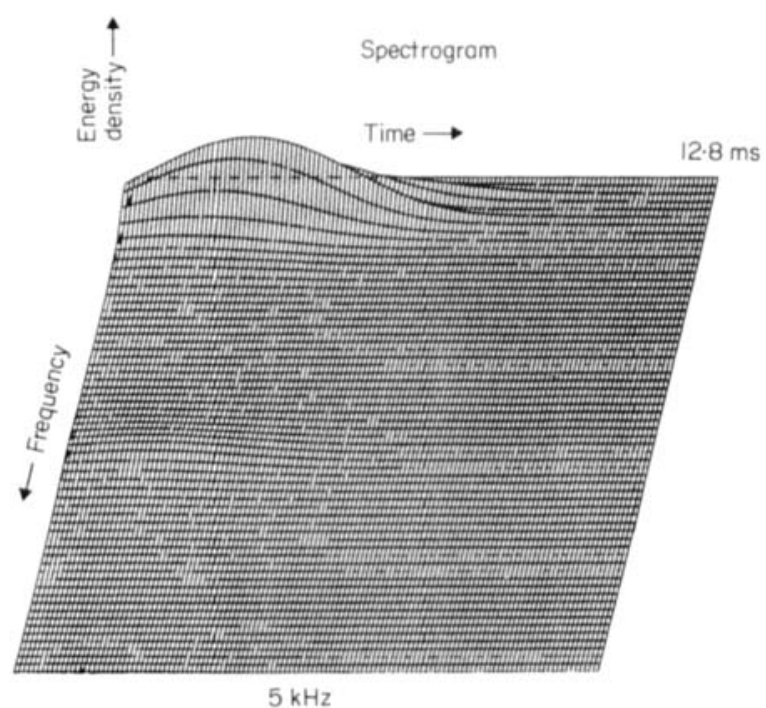

Fig. 4. Spectrogram three-dimensional graph of the same clicking as that shown in Figs 1-3. Note the lack of details, the paucity of high-frequency components, and the spread in time compared to the RID shown in Fig. 2.

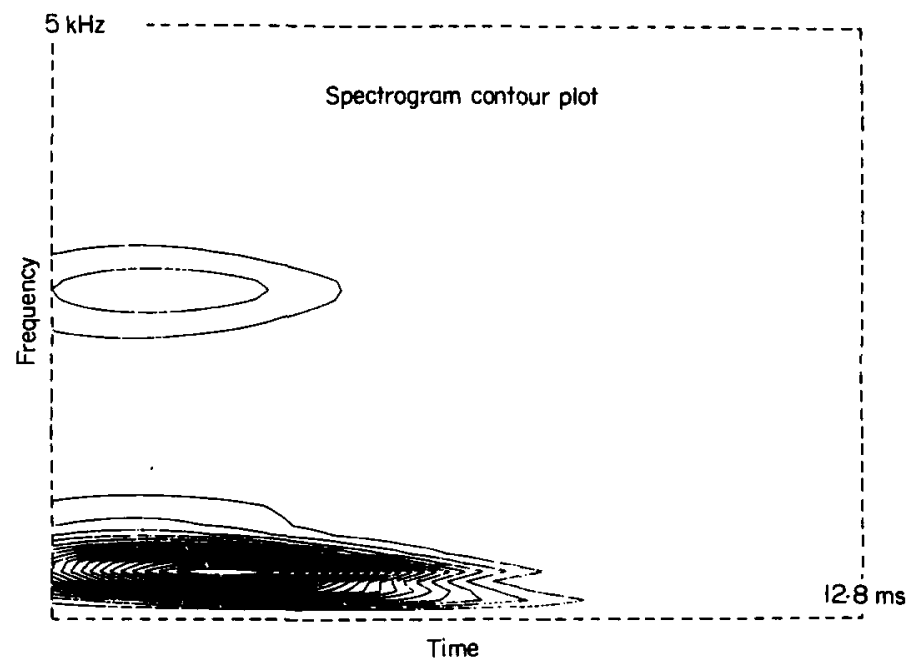

Fig. 5. Spectrogram contour plot of the same clicking as that shown in Figs 1-4. Note the lack of details, the paucity of high-frequency components, and the spread in time compared to Fig. 3.

compared to the spectrogram, is that the spectrogram but not the RID is using socalled sliding windows. The RID, like the Wigner distribution, is a true conjoint distribution. The disadvatages of using sliding windows for analysis of rapidly changing signals, such as TMJ sounds, are illustrated in Fig. 8. Conventional time-varying spectral analysis usually employs the spectrogram, which is formulated from the signal 


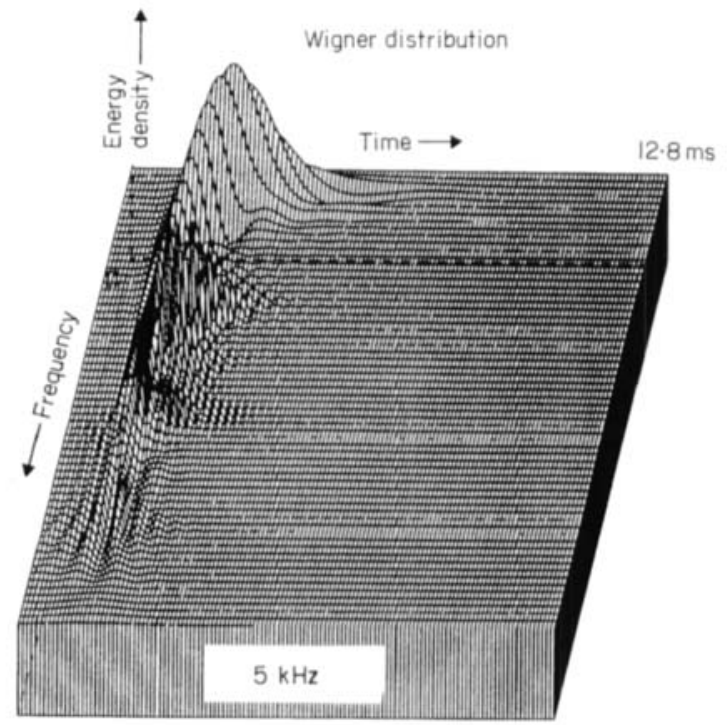

Fig. 6. Wigner distribution three-dimensional plot of the same clicking as that shown in Figs 1-5. Significant cross-terms are seen in the middle- and high-frequency ranges. They obscure the ridges that are so clearly visible in Fig. 2.

Wigner contour plot

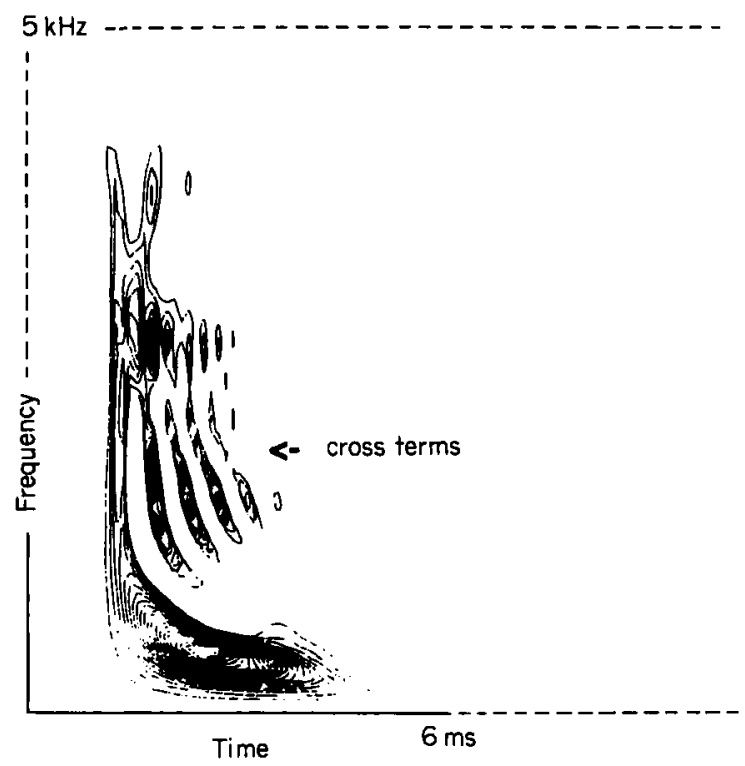

Fig. 7. Wigner distribution contour plot of the same clicking as that shown in Figs 1-6. As in Fig. 6, significant cross-terms are seen in the middle- and high-frequency ranges. Compare with Fig. 3.

$s(t)$ by taking a segment of $s(t)$ to form $s_{w}(t)$, a windowed portion of $s(t)$. Thus:

$$
s_{w}(t)=s(t) w(t)
$$



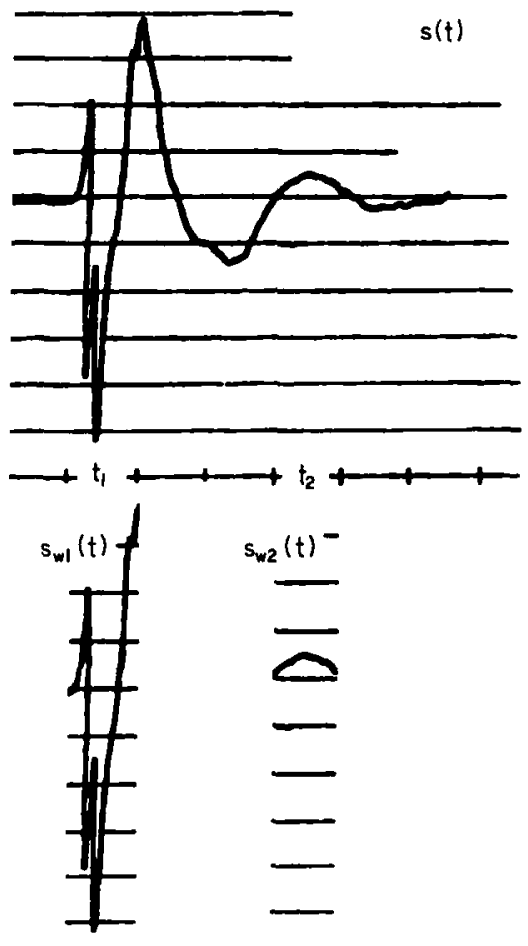

Window $1 \quad$ Window 2

Fig. 8. Illustration of problems encountered in windowing of a time-varying signal $s(t)$. The same clicking as that shown in Fig. 1 is displayed in the upper panel. Two window positions are illustrated in the lower panel, window 1 centred at $t_{1}$ and window 2 centred at $t_{2}$ (duration $2 \mathrm{~ms}$ ). Neither of the two signal parts captured, $S_{w 1}(t)$ or $S_{w 2}(t)$, are representative of the clicking sound. A short window duration is needed to locate the frequency components in time. That information is lost when using the spectrogram (see Figs 4 and 5), but not when using the RID (see Figs 2 and 3), if the window

where $w(t)$ is a window, with duration $d$, which may be unity valued from $-d / 2 \leqslant t \leqslant d / 2$ and have zero values outside this window. This is illustrated in Fig. 8. If it is assumed that the window is centred at $t_{1}$ or at $t_{2}$ then one first obtains the Fourier Transform of $\mathrm{s}_{\mathrm{w}}(\mathrm{t})$ :

$$
S_{w}(\omega)=\int_{-\infty}^{\infty} S_{w}(t) e^{-j \omega t} d t
$$

where $\omega$ is the frequency (radians $s^{-1}$ ). The power spectrum for that particular window position is then determined by the following expression:

$$
P_{w}(\omega)=\left|S_{w}(\omega)\right|^{2}
$$

It is obvious that neither $s_{w 1}(t)$ nor $s_{w_{2}}(t)$ is a good representation of $s(t)$ for the selected window duration $(2 \mathrm{~ms})$. If the duration of the window is increased to about $12 \mathrm{~ms}$, a determination of $P_{w}(\omega)$ can be made for the whole signal. Thus a power spectrum $P\left(\omega, t_{w}\right)$ can be found that provides an estimate of the frequency components captured within the whole clicking sound. However, 'this means that the time 
at which the frequency components act is imprecisely determined, since it is not known at exactly which point each frequency component is present. One must trade off large window durations, which resolve the frequency content within the window, against a precise determination of the time of action of the frequency components, which requires short-duration windows.

Biological signals usually exhibit components that are restricted in both duration and frequency, and thus often render spectrogram-based analysis ineffective. Other distributions, such as the newly developed reduced interference distribution (RID), utilize a different approach. The Wigner distribution, for example, is a true conjoint time-frequency distribution. The local autocorrelation of the signal may be found as:

$$
R(t, \tau)=s(t-\tau / 2) s^{*}(t+\tau / 2)
$$

where $s^{*}(t)$ is the complex conjugate of $s(t)$ which is often the analytical form of $s(t)$, which has both real and imaginary values. Then, the Wigner distribution is as follows:

$$
W(t, \omega)=\int_{-\infty}^{\infty} R(t, \tau) e^{-j \omega \tau} d \tau
$$

However, if $s(t)$ has more than one component, the product term of Equation (4) produces cross-terms between those components. For example, using a simple algebraic argument:

$$
(x+y)(x+y)=x^{2}+2 x y+y^{2}
$$

Here, $2 x y$ is a cross-term. It is, in fact, twice the size of the autoterms $x^{2}$ and $y^{2}$. The RID minimizes those cross-terms by applying a special weighting function to the local autocorrelation $\mathrm{R}(\mathrm{t}, \mathrm{\tau})$ so that the components, the cross-terms of components separated in time and frequency, are considerably reduced. This is accomplished by convolving $\mathbf{R}(t, \tau)$ in time (filtering) with a specially designed kernel, so that:

$$
R_{R I D}(t, \tau)=R(t, \tau){ }^{*} k(t, \tau)
$$

and then:

$$
\operatorname{RID}(t, \omega)=\int_{-\infty}^{\infty} R_{R I D}(t, \tau) e^{-j \omega \tau} d \tau
$$

In practice, $s(n)$ is a digitally sampled sequence obtained from $s(t)$, and computation is carried out using the Fast Fourier Transform (FFT). The window is not of infinite duration, but can be much larger than that for the spectrogram.

Aliased, or false lower frequency components, may appear in analogue data that are reconstructed from the original data acquired at an insufficient sampling rate. It is well known that the sampling rate must be twice as high as the highest frequency component in the sampled signal. The microphone itself provides significant protection against aliasing in this case, due to its high-frequency roll-off at $1200 \mathrm{~Hz}$. Subsequent recordings will, however, be made with microphones with a larger range of frequency response, since our results confirm the findings of Gay et al. (1987), that distinct components occur in the TMJ sounds well above $1200 \mathrm{~Hz}$. The positioning of the microphone on the forehead was suitable for comparison of different types of $t-f$ distribution using only one microphone, but in future clinical applications multichannel recordings from other sites will be tested (Watt, 1966).

The conclusion of Gay and Bertolami (1987), that spectral analysis has limited 
application in the differential diagnosis of TMJ disorders is, in our opinion, premature. The spectrogram may not be useful, particularly in the case of long analysis window duration. However, true conjoint $t$ - $f$ distributions, which have several advantages over spectrograms, appear to be well worth testing in classification of TMJ sounds.

By using the RID, patterns of time-frequency distributions of TMJ sounds may be related to other clinical findings, and diagnoses made by specialists involved in the management of temporomandibular disorders. However, a classification and correlation with other signs of various TM disorders requires the collection and analysis of data from large groups of healthy subjects and patients with various types of diagnosed TM disorders. This approach is now being tested by our research group.

\section{Acknowledgements}

This study was supported by awards from the Office of the Vice President of Research, The University of Michigan, and from the Office of Naval Research, Contract No. N00014-90-J-1654.

\section{References}

Boashash, B. (1991) Time-frequency analysis. In: Advances in Spectrum Estimation (ed. S. Haykin). Prentice-Hall, New Jersey.

Chol, H.I. \& WiLliams, W.J. (1989) Improved time-frequency representation of multicomponent signals using exponential kernels. The Institute of Electrical and Electronic Engineers. Transactions on Acoustics, Speech and Signal Processing. 37, 862.

COHEN, L. (1989) Time-frequency distributions. IEEE Proceedings, 77, 941.

EKENSTEN, B. (1952) Phonograms of anomalies of the temporomandibular joint in motion. Odontologisk Tidskrift, 60, 235.

Eriksson, L., Westesson, P-L. \& Rohlin, M. (1985) Temporomandibular joint sounds in patients with disc displacement. International Journal of Oral Surgery, 14, 428.

FARRAR, W.B. (1978) Characteristics of the condylar path in internal derangements of the TMJ. Journal of Prosthetic Dentistry, 39, 319.

Gay, T., Bertolamı, C.N., Donoff, R.B., Ketrh, D.A. \& Kelly, J.P. (1987) The acoustical characteristics of the normal and abnormal temporomandibular joint. Journal of Oral and Maxillofacial Surgery, 45, 397.

GAY, T. \& Bertolami, C.N. (1987) The spectral properties of temporomandibular joint sounds. Journal of Dental Research, 66, 1189.

GAY, T. \& Bertolami, C.N. (1988) The acoustical characteristics of the normal temporomandibular joint. Journal of Dental Research, 67, 56.

IsBerG, A., WIDMalm, S.E. \& Ivarsson, R. (1985) Clinical, radiographical and electromyographical study of patients with internal derangement of the temporomandibular joint. American Journal of Orthodontics, 88, 453.

Oster, G., KatzberG, R.W., Tallents, R.H., Morris, T.W., Bartholomew, J., Miller, T.L. \& Hayakawa, K. (1984) Characterization of temporomandibular joint sounds. Oral Surgery, Oral Medicine and Oral Pathology, 58, 10.

OUELLETTE, P.L. (1974) TMJ sound prints: electronic auscultation and sonagraphic audiospectral analysis of the temporomandibular joint. Journal of the American Dental Association, 89, 623.

RamfJord, S. \& ASh, M.M. JR (1983) Occlusion, 3rd edn, p. 244. Saunders, Philadelphia.

Roberts, C.A., Tallents, R.H., Katzberg, R.W., Sanchez-Woodworth, R.E., Manzione, J.V., Espeland, M.A. \& Handelman, S.L. (1986) Clinical and arthrographic evaluation of temporomandibular joint sounds. Oral Surgery, Oral Medicine and Oral Pathology, 62, 373.

WATt, D.M. (1966) Clinical applications of gnathosonics. Journal of Prosthetic Dentistry, 16, 83.

Widmalm, S.E. \& HedegÅRD, B. (1974) An apparatus for the synchronous registration of EMG activity in jaw muscles and of vibrations in the masticatory system. Journal of Oral Rehabilitation, $1,183$.

Widmalm, S.E. \& LaRSson, E.M. (1982) A new method for recording temporomandibular joint sounds and electrical jaw muscle activity in relation to jaw-opening degree. Acta Odontologica 
Scandinavica, 40, 429.

WiDMER, C.G. (1989) Temporomandibular joint sounds: a critique of techniques for recording and analysis. Journal of Craniomandibular Disorders: Facial and Oral Pain, 3, 213.

Williams, W.J. \& Jgong, J. (1990) New time-frequency distributions for the analysis of multicomponent signals. Advanced Algorithms and Architectures for Signal Processing, IV. SPIE, 1152, 483. 